\title{
Distribuição vertical do cavalo-marinho Hippocampus reidi Ginsburg, 1933 na região de Arraial do Cabo, Rio de Janeiro, Brasil
}

\author{
Viviane Martins de Oliveira ${ }^{1}$ \\ Natalie Villar Freret-Meurer ${ }^{2,3 *}$ \\ ${ }^{1}$ Universidade Federal Fluminense \\ Avenida General Milton Tavares de Souza, s/n, CEP 24210-346, Niterói - RJ, Brasil \\ ${ }^{2}$ Universidade do Estado do Rio de Janeiro \\ Avenida São Francisco Xavier, 524, CEP 20550-013, Rio de Janeiro - RJ, Brasil \\ ${ }^{3}$ Universidade Santa Úrsula \\ Rua Jornalista Orlando Dantas, 59, CEP 22231- 010, Rio de Janeiro - RJ, Brasil \\ * Autor para correspondência \\ nataliefreret@yahoo.com.br
}

Submetido em 06/05/2011

Aceito para publicação em 27/02/2012

\section{Resumo}

Os cavalos-marinhos são encontrados por todo o mundo, em águas costeiras tropicais e temperadas. Este trabalho teve como objetivo caracterizar as populações de Hippocampus reidi em diferentes categorias de profundidade e hidrodinamismo em Arraial do Cabo, Rio de Janeiro. O estudo foi desenvolvido utilizando mergulho autônomo, sendo realizadas transecções de $20 \mathrm{~m}$ x $5 \mathrm{~m}$ em dois estratos de profundidade: raso, entre 0 e $5 \mathrm{~m}$ e fundo, entre 5 e $10 \mathrm{~m}$. Através de censos visuais, os indivíduos foram identificados quanto à espécie, ao sexo e tamanho (height - HT). A densidade de $H$. reidi variou com a profundidade e o hidrodinamismo. A maior profundidade média foi encontrada na Ilha de Cabo Frio - Ponta do Anequin, já a menor foi encontrada na Praia do Forno - Ponta D'água. Não há diferença significativa no tamanho dos indivíduos e nem na razão sexual entre as categorias de profundidade. A razão sexual de $H$. reidi na população de Arraial do Cabo foi de 1:1. Os resultados obtidos indicaram que as características populacionais são semelhantes entre os dois estratos de profundidade.

Palavras-chave: Características populacionais; Hidrodinamismo; Profundidade; Syngnathidae

\section{Abstract}

Vertical distribution of the seahorse Hippocampus reidi Ginsburg, 1933 in the Arraial do Cabo region, Rio de Janeiro, Brazil. Seahorses are found all over the world, in tropical and temperate coastal waters. The main aim of this study was to characterize the Hippocampus reidi populations in different depth and hydrodynamism categories in Arraial do Cabo, Rio de Janeiro state. The study was developed through scuba diving, with transects of $20 \mathrm{~m}$ x $5 \mathrm{~m}$ in two different depth layers: shallow, between 0 and $5 \mathrm{~m}$, and deep, between 5 and $10 \mathrm{~m}$. Through visual surveys, individuals were identified according to species, sex, and size (height - HT). The density of $H$. reidi varied according to depth and hydrodynamism. The highest mean depth was found in the Cabo Frio Island - Ponta do Anequin, as the lowest depth was found in Praia do Forno - Ponta D'água. There is no significant 
difference in the size of individuals nor in the sex ratio between the depth categories. The sex ratio of $H$. reidi in the population of Arraial do Cabo was 1:1. The results obtained indicated that the population characteristics are similar between the two depth layers.

Key words: Depth; Hydrodynamism; Population characteristics; Syngnathidae

\section{Introdução}

Os peixes recifais incluem peixes de águas rasas $(<100 \mathrm{~m})$ tropicais e subtropicais, bentônicos ou bentopelágicos, que vivem numa forte associação com substratos de coral, algas, ou recifes rochosos ou ocupam a areia adjacente ao substrato (FLOETER et al., 2008). Possuem uma distribuição espacial dependente da dispersão de ovos e larvas por correntes oceânicas, uma vez que estes peixes são relativamente sedentários durante a fase adulta (LEIS, 1991; VICTOR, 1991).

A distribuição desses organismos está diretamente relacionada a variáveis como temperatura, luz, salinidade, nutrientes e outros fatores que interagem produzindo habitats distintos no ambiente marinho. Embora seja possível isolar cada variável e considerar a influência de cada uma sobre as populações separadamente, os peixes devem estar funcionalmente adaptados a um conjunto de variáveis ambientais. Para se entender essas adaptações e a dinâmica dos ecossistemas marinhos é essencial conhecer os fatores associados a esses ambientes (SOARES-GOMES; FIGUEIREDO, 2002).

Um dos fatores que pode afetar a ocupação do habitat é exposição às ondas no local. A exposição às ondas é representada pelo grau de movimentação das águas e é um importante fator que interfere na ecologia de ambientes de recifes rochosos, uma vez que influencia as condições químicas e físicas da água, bem como os processos biológicos como alimentação e reprodução (LEWIS, 1968).

O padrão de distribuição dos peixes nos ambientes recifais é fortemente relacionado ao padrão de distribuição vertical dos organismos bênticos. Algumas pesquisas descrevem distintas associações de peixes em diferentes zonações com vários tipos de substrato e complexidade em ambientes tropicais e temperados (EBELING; HIXON, 1991; HOLBROOK et al., 1990).
Existem diversas espécies de cavalos-marinhos em recifes de regiões tropicais e sub-tropicais de todo o mundo Esse animais são representados apenas pelo gênero Hippocampus Rafinesque, 1810, pertencendo à família Syngnathidae. Estes peixes são encontrados normalmente em águas rasas, porém alguns indivíduos têm sido registrados em profundidades entre 40 e $100 \mathrm{~m}$ (FOSTER; VINCENT, 2004). Algumas espécies podem ser encontradas em diferentes tipos de microhabitats (ex.: algas, briozoários, esponjas), já outras espécies podem viver estritamente associadas a um tipo de microhabitat em particular (CURTIS et al., 2004). Algumas espécies de cavalos-marinhos mudam de habitat e profundidade na medida em que crescem. As diferenças de ocupação espacial sugerem que as oportunidades de alimento ou risco de predação são muito variados entre as espécies ou que estes animais não determinam a escolha de seu habitat. Entre os indivíduos adultos, as classes de tamanho apresentam diferenças na ocupação do habitat (FOSTER; VINCENT, 2004). Informações sobre o uso do habitat, em diferentes escalas espaciais, são uma importante ferramenta para se caracterizar a distribuição dos cavalos-marinhos nesses ambientes, auxiliando na elaboração de medidas conservacionistas para reduzir o impacto sobre o habitat e a população estudada. Mudanças na amplitude da distribuição, proporção sexual ou atividade reprodutiva em animais da população podem ser usadas para avaliar os efeitos das estratégias de manejo ou eventos de distúrbio (CURTIS et al., 2004; ROSA et al., 2007).

No Brasil existem apenas duas espécies de cavalosmarinhos, sendo elas Hippocampus reidi Ginsburg, 1933 e Hippocampus erectus Perry, 1810. A espécie H. reidi é a mais abundante no Brasil (ROSA et al., 2002), entretanto encontra-se na Lista de Espécies Ameaçadas do Estado do Rio de Janeiro com o status de "vulnerável" e na Lista Vermelha da União Internacional para a Conservação da Natureza (IUCN, 2006) como "dados deficientes". Ela distribui-se desde Cabo Hatteras, Estados Unidos, à 
Santa Catarina, Brasil, e no Golfo do México (LOURIE et al., 1999). Existem registros de ocorrência de $10 \mathrm{~cm}$ de profundidade (ROSA et al., 2002) a 55m (VARI, 1982).

O presente trabalho tem como objetivo caracterizar a população do cavalo-marinho brasileiro Hippocampus reidi de Arraial do Cabo, dando ênfase aos seguintes aspectos: composição de jovens e adultos, razão sexual, profundidade e o hidrodinamismo da área de ocorrência.

\section{Material e Métodos}

\section{Área de estudo}

O estudo foi realizado em seis pontos selecionados de acordo com a viabilidade logística no município de

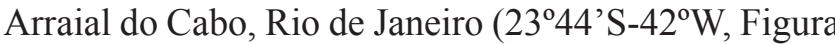
1) (LAGES, 2003). Os pontos selecionados possuem características diferentes entre si e o grau de exposição às ondas pode variar de acordo com as condições climáticas.

FIGURA 1: Mapa da área de estudo Arraial do Cabo - RJ: 1)Saco do Cardeiro; 2) Ilha de Porcos - Ponta Sul; 3) Ilha de Porcos - Saltador; 4) Ilha de Cabo Frio - Saco da Abobrinha; 5) Ilha de Cabo Frio - Ponta do Anequim 6) Praia do Forno- Ponta d'água. (Figura adaptada de FERREIRA et al., 1998).

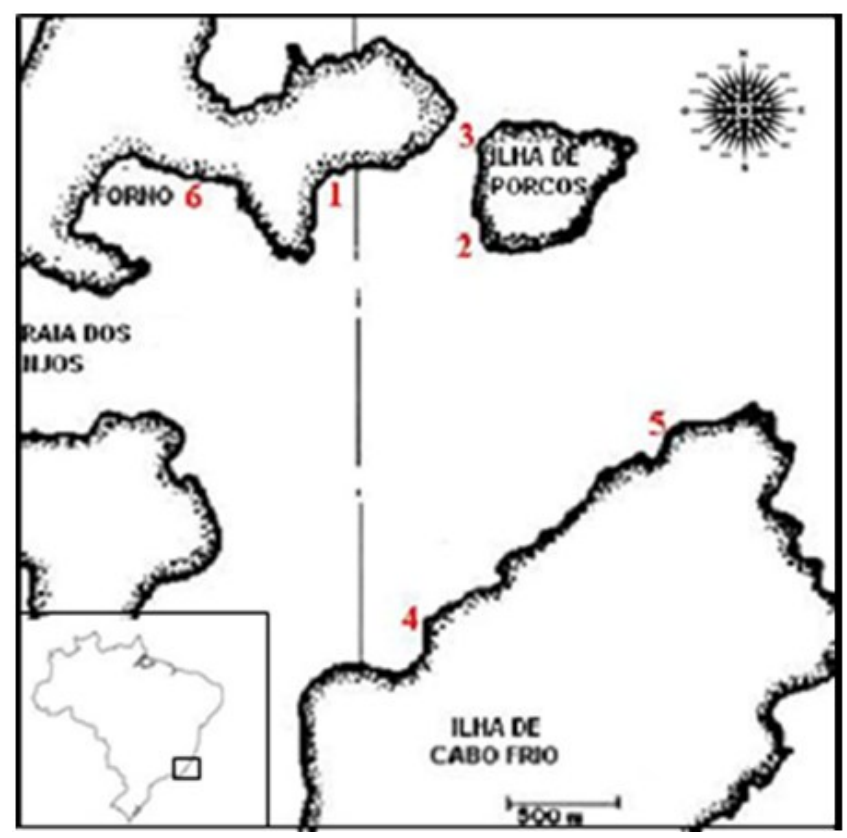

Arraial do cabo é caracterizado pela ocorrência de ressurgência. Tal evento está associado com o regime de vento local nos períodos de primavera e verão e com a batimetria. Entretanto, a área de estudo é afetada somente por períodos curtos e, geralmente, em áreas profundas (LAGES, 2003).

O Saco do Cardeiro é um local de mergulho raso, e em geral de mar calmo. A profundidade junto ao costão varia de 6 a 10m, o fundo é caracterizado por rochas cobertas pelo zoantídeo Palythoa caribaeorum Duchassaing \& Michelotti, 1860; gorgônias e colônias de esponjas, Aplysina sp. Nardo (1834). Os dois pontos da Ilha de Porcos possuem fundo similar ao do Saco do Cardeiro, porém um pouco mais profundo, chegando a $15 \mathrm{~m}$. É um ponto protegido quando está soprando o vento nordeste, predominante na região (LAGES, 2003). A Ponta do Anequim é uma enseada razoavelmente abrigada localizada na Ilha de Cabo Frio. O local fica bem protegido de ventos sul e sudoeste, mas aumenta seu hidrodinamismo com ventos fortes de nordeste. A profundidade média é de $10 \mathrm{~m}$, com máximo de $15 \mathrm{~m}$. O substrato na Ponta do Anequim é predominantemente coberto por gorgônias. O Saco da Abobrinha também localizado na Ilha de Cabo Frio possui características semelhantes à Ponta do Anequin, porém com menor profundidade, cerca de 6 a 10m, e uma abundância da esponja Aplysina sp. Nardo (1834) (FERREIRA et al., 2004). A Ponta d'água localiza-se próxima à Praia do Forno. É uma área relativamente rasa, mas pode chegar a $14 \mathrm{~m}$ de profundidade em alguns pontos, e abrigada, formando uma pequena enseada. $\mathrm{O}$ recife rochoso dessa área apresenta grande quantidade da esponja Aplysina sp.

\section{Metodologia}

O estudo foi realizado com observações in situ entre o período de 11 de agosto de 2007 a 27 de abril de 2008 utilizando mergulho autônomo. Foram realizadas oito transecções aleatórias paralelas à linha da costa de $20 \mathrm{~m}$ $\mathrm{x} 5 \mathrm{~m}$, totalizando uma área de $100 \mathrm{~m}^{2}$, entre 0 e $5 \mathrm{~m}$ e oito transecções aleatórias paralelas à linha da costa, com as mesmas dimensões, entre 5 e $10 \mathrm{~m}$, contando a partir de 5,1 m. Totalizando 16 transecções, por dia de amostra em cada um dos seis pontos amostrais selecionados no município de Arraial do Cabo, Rio de Janeiro. Sendo 
feitos dois pontos amostrais por dia de amostra, com total de 32 transecções.

Ao longo das transecções foi utilizado o método de censo visual para estimar a densidade, distribuição vertical, proporção de jovens e adultos e razão sexual das populações de cavalos-marinhos.

A abundância foi estimada a partir da contagem do número de indivíduos encontrados ao longo das transecções e, posteriormente, foi calculada a densidade (número de indivíduos/área). Todos os exemplares foram identificados em campo de acordo com Lourie et al. (1999) e Figueiredo e Menezes (1980), em que o tamanho do focinho é maior que à distância da margem posterior da órbita à abertura branquial e pelo padrão de coloração com listras pálidas dorsais e pontos escuros pelo corpo. A profundidade na qual cada indivíduo foi encontrado foi aferida através de um profundímetro da marca Cressi. Os estágios juvenil e adulto foram determinados em todos os indivíduos observados a partir de sua altura (height-HT), que compreende a distância vertical entre o topo da coroa e o fim da cauda preênsil esticada (LOURIE, 2003) e, de acordo com Silveira (2005). Adultos foram definidos como de altura superior a $10 \mathrm{~cm}$, enquanto jovens foram definidos como de altura inferior a $10 \mathrm{~cm}$. A identificação do sexo dos indivíduos foi realizada pela determinação da presença ou ausência da bolsa incubadora, uma vez que a bolsa é encontrada somente nos machos e se localiza na região ventral do animal. Todos os dados foram registrados em uma prancheta subaquática.

O grau de exposição às ondas foi definido como uma variável categórica, baseado nas observações visuais feitas in loco durante o período de amostragem. O grau de exposição às ondas foi ranqueado em (1) calmo, quando o mar encontra-se plano ou com pequenas ondulações; (2) crespo, com ondulações largas; (3) moderado, com muitas ondulações com formação de espuma e (4) agitado, com grandes ondas de 2-3m, segundo a classificação de Curtis et al. (2004).

O número de machos e fêmeas por profundidade, assim como a diferença de densidade entre os dois estratos de profundidade foi avaliada através do teste t. A proporção de jovens e adultos por profundidade e a relação entre o número de indivíduos nos dois estratos e o grau intensidade das ondas foram avaliadas através do Teste Chi-Quadrado. A análise de teste $t$ seguiu as premissas de normalidade e homogeneidade das variâncias (ZAR, 1999). Os valores médios foram expressos pela média \pm desvio padrão.

\section{Resultados}

Nas 560 transecções realizadas durante o período amostral foi encontrada densidade média de 0,01 \pm 0,016 indivíduos. $\mathrm{m}^{-2}$. O ponto amostral que apresentou a maior densidade individual foi na Praia do Forno onde a densidade média encontrada foi de 0,044 \pm 0,008 indivíduos. $\mathrm{m}^{-2}$. As áreas de menor densidade média foram na Ilha de Cabo Frio com 0,002 $\pm 0,001$ indivíduos.m $\mathrm{m}^{-2}$ no Saco da Abobrinha e 0,001 $\pm 0,0024$ indivíduos. $\mathrm{m}^{-2}$ na Ponta do Anequim.

Foram registrados indivíduos a uma profundidade mínima de $1 \mathrm{~m}$ e máxima de $8 \mathrm{~m}$. A profundidade média onde foram encontrados os cavalos-marinhos foi de 4,97 $\pm 1,51 \mathrm{~m}$. A maior profundidade média de ocorrência de cavalos-marinhos foi encontrada na Ilha de Cabo Frio Ponta do Anequim, com $6,5 \pm 0,70 \mathrm{~m}$, seguida pela Ilha de Porcos - Ponta Sul, com uma profundidade média de $6,27 \pm 0,25 \mathrm{~m}$. No Saco do Cardeiro a profundidade média foi de 5,44 $\pm 1,30 \mathrm{~m}$. Já a Ilha de Cabo Frio - Saco da Abobrinha a profundidade média encontrada foi de $5 \pm 0 \mathrm{~m}$. Na Ilha de Porcos - Saltador foi encontrada uma profundidade média de $4,17 \pm 1,52 \mathrm{~m}$. A menor profundidade média foi registrada na Praia do Forno, $3,57 \pm 1,27 \mathrm{~m}$. A profundidade média de $H$. reidi acima dos $5 \mathrm{~m}$ foi de $3,94 \pm 2,00 \mathrm{~m}$ e abaixo de $5 \mathrm{~m}$ foi de 6,39 $\pm 1,71 \mathrm{~m}$. A densidade média no estrato acima de $5 \mathrm{~m}$ foi de $0,078 \pm 0,07$ indivíduos. $\mathrm{m}^{-2}$ e abaixo de $5 \mathrm{~m}$ a densidade média foi de $0,060 \pm 0,07$ indivíduos. $m^{-2}$ não havendo diferença significativa entre a densidade de ambos estratos $(p=0,430 ; \mathrm{U}=229,0 ; \mathrm{gl}=22)$.

A relação entre a intensidade das ondas e a distribuição dos cavalos-marinhos revelou uma correlação significativa entre o gradiente de profundidade e intensidade das ondas ( $\left.p=0,001 ; \chi^{2}=9,702\right)$ (Figura 2 ), mostrando que quanto maior a intensidade das ondas, maior é a profundidade de ocorrência do cavalo-marinho. 
Foram encontrados apenas sete jovens, sendo quatro a cima de $5 \mathrm{~m}$ de profundidade e três abaixo de $5 \mathrm{~m}$. Não foi encontrada nenhuma associação entre a proporção de jovens e adultos e ambos os estratos de profundidade $\left(p=0,085 ; \chi^{2}=0,031\right)$ (Tabela 1$)$. A média de tamanho dos cavalos-marinhos foi de $11,06 \pm 2,00 \mathrm{~cm}$ acima dos $5 \mathrm{~m}$ e de $11,83 \pm 1,72 \mathrm{~cm}$ abaixo dos $5 \mathrm{~m}$. O valor médio da profundidade de machos e fêmeas também não apresentou grande variação, sendo de $5,11 \pm 1,42 \mathrm{~m}$ para as fêmeas e de 4,93 $\pm 1,71 \mathrm{~m}$ para os machos. Nos dois estratos este valor também não apresentou grande variação, sendo de $4,17 \pm 0,77 \mathrm{~m}$ para as fêmeas e de 3,64 $\pm 1,30 \mathrm{~m}$ para os machos acima dos $5 \mathrm{~m}$ e de $6,4 \pm 1,02 \mathrm{~m}$ para as fêmeas e $6,38 \pm 0,47 \mathrm{~m}$ para os machos abaixo dos $5 \mathrm{~m}$ de profundidade. Portanto machos e fêmeas possuem padrões semelhantes de distribuição vertical. A razão sexual total dos cavalos-marinhos de Arraial do Cabo foi de 19 de machos pra 19 fêmeas, sendo uma proporção equivalente de $1: 1$, porém cada população apresentou razão sexual variada. Nas populações do Saco do Cardeiro e da Praia do Forno, foram encontradas mais fêmeas que machos (11:7 e 4:3, respectivamente). Já Na Ponta Sul e no Saltador, ambos na Ilha de Porcos, foram encontrados mais machos que fềmeas (2:1 e 5:2, respectivamente). Nas demais áreas, foi encontrado apenas um dos sexos (Tabela 2).
FIGURA 2: Relação entre o gradiente de profundidade de ocorrência de $H$. reidi e a intensidade das ondas observada em Arraial do Cabo, RJ: 1) Calmo, 2) Moderado, 3) Crespo e 4) Agitado.

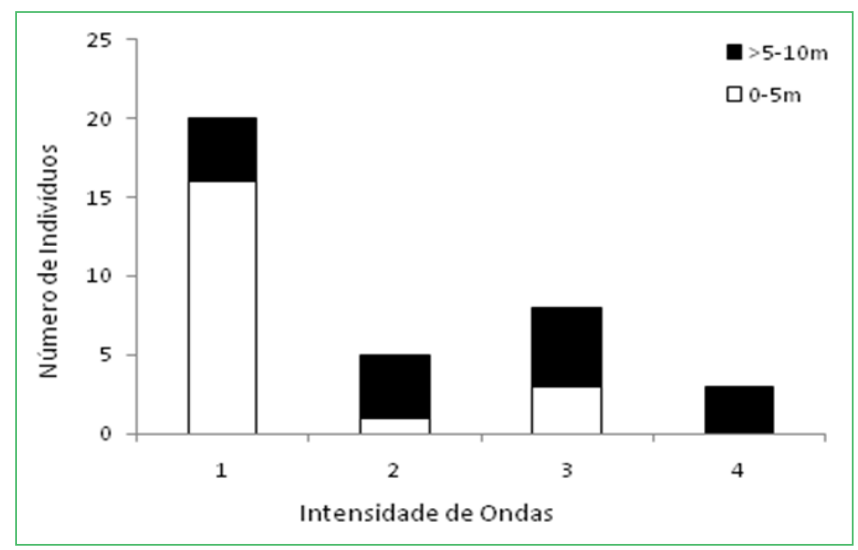

TABELA 1: Número de indivíduos de cavalos-marinhos da espécie $H$. reidi em relação à classe de comprimento e ao estrato de profundidade de ocorrência.

\begin{tabular}{|c|c|c|c|c|c|c|c|c|c|}
\hline $\begin{array}{r}\text { Classe de } \\
\text { comprimento }\end{array}$ & 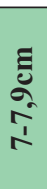 & $\begin{array}{l}\Xi \\
\text { हूँ } \\
\infty \\
\infty \\
\infty\end{array}$ & $\begin{array}{l}\text { हूँ } \\
\text { ă } \\
\text { à }\end{array}$ & 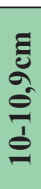 & 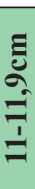 & 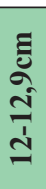 & $\begin{array}{l}\Xi \\
\text { हू } \\
\text { ले } \\
\stackrel{1}{n}\end{array}$ & $\begin{array}{l}\Xi \\
\text { J } \\
\text { Jे } \\
\dot{J} \\
\dot{J}\end{array}$ & $\begin{array}{l}\text { हू } \\
\text { กू } \\
\frac{1}{7} \\
\end{array}$ \\
\hline $0-5 m$ & 2 & 1 & 1 & 4 & 5 & 4 & 2 & 0 & 1 \\
\hline$>5-10 \mathrm{~m}$ & 0 & 0 & 3 & 2 & 3 & 2 & 5 & 1 & 0 \\
\hline
\end{tabular}

TABELA 2: Comprimento médio $(\mathrm{cm})$, número de machos e fêmeas, densidade média $\left(\mathrm{m}^{-2}\right)$ e profundidade média $(\mathrm{m})$ em cada ponto amostral.

\begin{tabular}{l|c|c|c|c|c|c}
\hline Ponto Amostral & $\mathbf{N}$ & $\begin{array}{c}\text { Altura médio } \\
(\mathbf{\pm} \mathbf{d}) \mathbf{c m}\end{array}$ & machos & fêmeas & $\begin{array}{c}\text { Densidade média } \\
(\mathbf{\pm d p}) \mathbf{~ m}^{-\mathbf{2}}\end{array}$ & $\begin{array}{c}\text { Profundidade média } \\
( \pm \mathbf{d p}) \mathbf{~ m}\end{array}$ \\
\hline Saco do Cardeiro & 18 & $11,97 \pm 1,54$ & 7 & 11 & $0,012 \pm 0,004$ & $5,44 \pm 1,30$ \\
\hline Ponta Sul & 3 & $12,6 \pm 0,69$ & 2 & 1 & $0,004 \pm 0,002$ & $6,27 \pm 0,25$ \\
\hline Saltador & 7 & $14,97 \pm 4,69$ & 5 & 2 & $0,005 \pm 0,003$ & $4,17 \pm 1,52$ \\
\hline Saco da Abobrinha & 1 & 7,9 & 0 & 1 & $0,002 \pm 0,001$ & $5 \pm 0$ \\
\hline Ponta do Anequim & 2 & $9,75 \pm 1,06$ & 2 & 0 & $0,001 \pm 0,0024$ & $6,5 \pm 0,70$ \\
\hline Praia do Forno & 7 & $9,73 \pm 1,80$ & 3 & 4 & $0,044 \pm 0,008$ & $3,57 \pm 1,27$ \\
\hline TOTAL & 38 & $11,93 \pm 2,94$ & 19 & 19 & $0,01 \pm 0,016$ & $5,02 \pm 1,52$ \\
\hline
\end{tabular}




\section{Discussão}

Foi encontrada uma baixa densidade de cavalosmarinhos na região estudada de Arraial do Cabo. Essa baixa densidade populacional encontrada para $H$. reidi também foi descrita por Lourie (1999), Foster e Vincent (2004), Lourie et al. (2004), Rosa et al. (2007), FreretMeurer e Andreata (2008) e Freret-Meurer et al. (em preparação) e caracteriza um padrão de distribuição em manchas. As densidades estimadas para cavalosmarinhos geralmente são mais baixas em pesquisas de transecção aleatória, como utilizada neste trabalho, que cobrem uma área menor em linha reta; do que em pesquisas de rede ou grade ( grid), que, por cobrir uma área melhor distribuída, apresenta uma densidade populacional maior (FOSTER; VINCENT, 2004). Os estudos com grade, em geral trabalham com as manchas de cavalos-marinhos já localizadas, o que proporciona também esta elevada densidade.

A intensidade das ondas foi positivamente relacionada à profundidade de ocorrência dos cavalosmarinhos. Este fato era previsto, pois estes peixes têm adaptações específicas à vida bentônica, como a cauda preênsil que os permite agarrar-se ao substrato, auxiliando no forrageio, no descanso, além de favorecer ao melhor desempenho da camuflagem permanecendo imóveis juntos ao substrato. Sua locomoção também é limitada devido ao deslocamento ser realizado por movimentos ondulatórios da nadadeira dorsal (CONSI et al., 2001). Presume-se que uma área de forte intensidade não permite uma boa natação, nem fixação ou camuflagem fazendo com que estes animais prefiram deslocar-se para áreas mais profundas. Estes dados podem auxiliar na escolha de locais e métodos mais adequados para realizar trabalhos de levantamentos populacionais dessa espécie, sugerindo que áreas com maior intensidade de ondas e rasas não são habitats adequados para ocorrência de cavalos-marinhos. Enquanto que áreas com baixa intensidade de ondas, rasas ou mais profundas, formam um ambiente mais adequado para ocorrência dessa espécie. Neste caso, devem também ser levados em consideração outros fatores bióticos e abióticos que possam justificar a ocorrência ou não da espécie.
Não foi encontrada variação ontogenética na ocorrência de profundidade, uma vez que quatro jovens foram observados a cima de $5 \mathrm{~m}$ e outros três jovens, abaixo de $5 \mathrm{~m}$. O trabalho de Dauwe (1992) em ambientes recifais de Bonaire, nas Antilhas Holandesas, mostra que os indivíduos dessa espécie de cavalo-marinho mudam de habitat e profundidade à medida que crescem, havendo ocupação de indivíduos maiores em áreas mais profundas.

Foi encontrada razão sexual total de cavalosmarinhos de Arraial do Cabo igual para machos e fêmeas. Essa razão sexual encontrada corrobora com os resultados obtidos por Dauwe (1992), Rosa et al (2007) e Freret-Meurer e Andreata (2008), que também encontraram uma relação aproximada de machos e fêmeas de 1 para 1 . Assim como estudos sobre outras espécies de cavalos-marinhos como H. comes Cantor 1850, H. capensis Boulenger 1900 e H. gluttulatus Cuvier 1829, que também tiveram uma proporção equitativa de machos e fêmeas (PERANTE et al., 2002; BELL et al., 2003; CURTIS; VINCENT, 2006). Entretanto, para cada área analisada, foi encontrada razão sexual variada, havendo padrão em três grupos distintos: I) Praia do Forno e Saco do Cardeiro apresentaram mais fêmeas do que machos; II) Saltador e Ponta Sul apresentaram mais machos do que fêmeas e III) Saco da Abobrinha e Ponta do Anequim apresentaram ausência de um dos sexos, entretanto, complementares entre ambas as áreas. A proximidade geográfica entre as áreas de cada grupo pode ser um fator que explique a semelhança nessa composição populacional, sugerindo, que talvez cada grupo forme uma mancha com diferente estrutura. A variada razão sexual pode também indicar diferentes sistemas de acasalamento nessas populações, visto que há uma forte relação entre a razão sexual e o sistema de acasalamento de uma população (JONES; AVISE, 1997).

A distribuição geográfica da maioria das espécies de cavalo-marinho permanece mal definida e caracterizada (LOURIE et al., 1999; KUITER, 2001; CHOO; LIEW, 2003) e a maioria das espécies também se encontra em algum grau de ameaça ou deficiência de dados, portanto são necessários mais estudos referentes à distribuição e estrutura das populações de cavalos-marinhos para 
tornar possível qualquer forma de manejo e conservação das espécies.

\section{Agradecimentos}

Este trabalho contou com o apoio logístico da operadora de mergulho Dive Point.

\section{Referências}

BELL, E. M.; LOCKYEAR, J. F.; MCPHERSON, J. M.; MARSDEN, A. D.; VINCENT, A. C. J. The first field studies of an endangered South African seahorse, Hippocampus capensis. Environmental Biology of Fishes, New York, v. 67, p. 35-46, 2003.

CHOO, C. K.; LIEW, H. C. Spatial distribution, substrate assemblages and size composition of sea horses (Family Syngnathidae) in the coastal waters of Penninsular Malaysia. Journal of Marine Biology Association, Cambridge, v. 83, p. 271276, 2003.

CONSI, T. R.; SEIFERT, P. A.; TRIANTAFYLLOU, M. S.; EDELMAN, E. R. The dorsal fin engine of the seahorse (Hippocampus sp.). Journal of Morphology, Malden, v. 248, p. 80-97, 2001.

CURTIS, J.; MOREAU, M. A.; MARSDEN, D.; BELL, E.; MARTIN-SMITH, K.; SAMOILYS, M.; VINCENT, A. Underwater visual census for seahorse population assessments. Project Seahorse Technical Report, Vancouver, n. 8, p. 28, 2004.

CURTIS, J. M. R.; VINCENT, A. C. J. Life history of an unusual marine fish: survival, growth and movement patterns of Hippocampus guttulatus (Cuvier 1829). Journal of Fish Biology, Maiden, v. 68, p. 707-733, 2006.

DAUWE, B. Ecology of the seahorse Hippocampus reidi on the Bonaire coral reef (N.A.): habitat, reproduction and community interactions. 1992. 63 f. Dissertação (Mestrado) Rijksuniversiteit, Gröningen. 1992.

EBELING, A. W.; HIXON, M. A. Tropical and temperate reef fishes: a comparison of community structures. In: SALE, P. F. (Ed.). The ecology of fishes on coral reefs. San Diego: Academic Press, 1991. p. 509-563.

FERREIRA, C. E. L.; GONÇALVES, J. E. A.; COUTINHO R. Cascos de navios e plataformas como vetores na introdução de espécies exóticas. In: SILVA, J. S. V.; SOUZA, R. C. C. L. (Org.). Água de Lastro e Bioinvasão. Rio de Janeiro: Interciência, 2004. p. 143-153.

FIGUEIREDO, J. L.; MENEZES, N. Manual de peixes marinhos do Sudeste do Brasil III - Teleostei 2. São Paulo: Museu de Zoologia da Universidade de São Paulo, 1980. 90 p.

FLOETER, S. R.; ROCHA, L. A.; ROBERTSON, D. R.; JOVEUX, J. C.; SMITH-VANIZ, W. F.; WIRTZ, P.; EDWARDS, A. J.; BARREIROS, J. P.; FERREIRA, C. E. L.; GASPARINI, J. L.; BRITO, A.; FALCON, J. M.; BOWEN, B. W.; BERNARDI, G. Atlantic reef fish biogeography and Evolution. Journal of Biogeography, New York, v. 35, p. 22-47, 2008.
FOSTER, S. J.; VINCENT, A. C. J. Life history and ecology of seahorses: implications for conservation and management. Journal of Fish Biology, Maiden, v. 65, p. 1-61, 2004.

FRERET-MEURER, N. V.; ANDREATA, J. V. Field studies of a Brazilian seahorse population, Hippocampus reidi Ginsburg, 1933. Brazilian Archieves of Biology and Technology, Curitiba, v. 51, n. 4, p. 743-751, 2008.

HOLBROOK, S. J.; CARR, M. H.; SCHMITT, R. J.; COYER, J. A. Effect of Giant kelp on local abundance of reef fishes: the importance of ontogenetic resource requirements. Bulletin of Marine Science, Miami, v. 41, n. 1, p. 104-114, 1990.

IUCN - INTERNATIONAL UNION FOR CONSERVATION OF NATURE. Red List of Threatened Species. 2006. Disponível em $<$ http://www.iucnredlist.org/>. Acesso em: 5 maio 2007.

JONES, A. G.; AVISE, J. C. Microsatellite analysis of maternity and the mating system in the Gulf pipefish Syngnathus scovelli, a species with male pregnancy and sex-role reversal. Molecular Ecology, Vancouver, v. 6, p. 203-213, 1997.

KUITER, R. H. Revision of the Australian Seahorses of the Genus Hippocampus (Syngnathiformes: Syngnathidae) with Descriptions of Nine New Species. Records of the Australian Museum, Sidney, v. 53, 293340, 2001.

LAGES, B. G. Avaliação do potencial invasor do coral alcionáceo Stereonephthya aff. curvata (Nephtheidae - Alcyonacea) na Reserva Extrativista Marinha de Arraial do Cabo (RJ). 2003. 61 f. Dissertação (Mestrado em Biologia Marinha) - Universidade Federal Fluminense, Niterói. 2003.

LEIS, J. M. The pelagic stage of reef fishes: the larval biology of coral reef fishes. In: SALE, P. F. (Ed.). The ecology of fishes on coral reefs. San Diego: Academic Press, 1991. p. 183-230.

LEWIS, J. R. Water movement and their role in rocky shore ecology. Sarsia, Bergen, v. 34, p. 13-36, 1968.

LOURIE, S. Measuring seahorses. Project Seahorse Technical Report, Dordrecht, n. 4, p. 15, 2003.

LOURIE, S. A.; FOSTER, S. J.; COOPER, E. W. T.; VINCENT, A. C. J. A guide to the identification of seahorses. Washington: Project Seahorse and TRAFFIC North America, 2004. 114 p.

LOURIE, S. A.; VINCENT, A. C. J.; HALL, H. Seahorses: an identification guide to the world's species and their conservation. London: Project Seahorse, 1999. 214 p.

PERANTE, N. C.; PAJARO, M. G.; MEEUWIG, J. J.; VINCENT, A. C. J. Biology of a seahorse species, Hippocampus comes in the central Philippines. Journal of Fish Biology, Maiden, v. 60, p. 821837, 2002.

ROSA, I. L.; DIAS, T. L.; BAUM, J. K. Threatened fishes of the world: Hippocampus reidi Ginsburg, 1933 (Syngnathidae). Environmental Biology of Fishes, Dordrecht, v. 64, p. 378, 2002.

ROSA, I. L.; OLIVEIRA, T. P. R.; CASTRO, A. L. C; SOUZA MORAES, L. E.; XAVIER, J. H. A.; NOTTINGHAM, M. C.; DIAS, T. L. P.; BRUTO-COSTA, L. V.; ARAÚJO, M. E.; BIROLO, A. B.; MAI, A. C. G.; MONTEIRO-NETO, C. Population characteristics, space use and habitat associations of the seahorse Hippocampus reidi (Teleostei: Syngnathidae). Neotropical Ichthyology, Porto Alegre, v. 5, n. 3, p. 405-414, 2007.

SILVEIRA, R. B. Dinâmica populacional do cavalo-marinho Hippocampus reidi no manguezal de Maracaípe, Ipojuca, 
PE. 2005. 129 f. Tese (Doutorado em Zoologia) - Pontifícia Universidade Católica do Rio Grande do Sul, Porto Alegre, 2005.

SOARES-GOMES, A.; FIGUEIREDO, A. G. O. Ambiente Marinho. In: PEREIRA, R. C.; SOARES-GOMES, A. (Org.). Biologia Marinha. Rio de Janeiro: Interciência, 2002. p. 1-34.

VARI, R. P. Fishes of the Western North Atlantic, subfamily Hippocampinae. The seahorses. Marine Research Memoir, New Haven, v. 1, p. 173-189, 1982.

VICTOR, B. C. Settlement strategies and biogeography of reef fishes. In: SALE, P.F. (Ed.). The ecology of fishes on coral reefs. San Diego: Academic Press, 1991. p. 231-260.

ZAR, J. H. Biostatistical analysis. 4 ed. New Jersey: Prentice Hall, 1999. $662 \mathrm{p}$. 